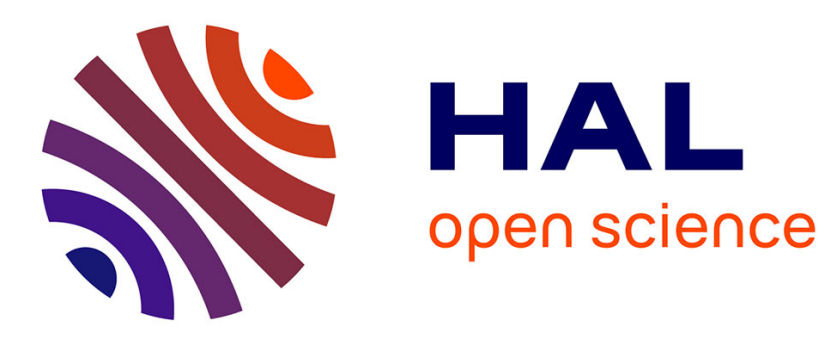

\title{
Decentralized Simultaneous Energy and Information Transmission in Multiple Access Channels
}

\author{
Selma Belhadj Amor, Samir M. Perlaza
}

\section{To cite this version:}

Selma Belhadj Amor, Samir M. Perlaza. Decentralized Simultaneous Energy and Information Transmission in Multiple Access Channels. 50th Annual Conference on Information Sciences and Systems (CISS), Princeton University - Department of Electrical Engineering and Technical Co-sponsorship with IEEE Information Theory Society, Mar 2016, Princeton, NJ, United States. hal-01262793

\section{HAL Id: hal-01262793 \\ https://hal.science/hal-01262793}

Submitted on 27 Jan 2016

HAL is a multi-disciplinary open access archive for the deposit and dissemination of scientific research documents, whether they are published or not. The documents may come from teaching and research institutions in France or abroad, or from public or private research centers.
L'archive ouverte pluridisciplinaire HAL, est destinée au dépôt et à la diffusion de documents scientifiques de niveau recherche, publiés ou non, émanant des établissements d'enseignement et de recherche français ou étrangers, des laboratoires publics ou privés. 


\title{
Decentralized Simultaneous Energy and Information Transmission in Multiple Access Channels
}

\author{
Selma Belhadj Amor and Samir M. Perlaza
}

\begin{abstract}
In this paper, the fundamental limits of decentralized simultaneous information and energy transmission in the twouser Gaussian multiple access channel (G-MAC) are fully characterized for the case in which a minimum energy transmission rate $b$ is required for successful decoding. All the achievable and stable information-energy transmission rate tuples $\left(R_{1}, R_{2}, B\right)$ are identified. $R_{1}$ and $R_{2}$ are in bits per channel use measured at the receiver and $B$ is in energy units per channel use measured at an energy-harvester (EH). Stability is considered in the sense of an $\eta$-Nash equilibrium (NE), with $\eta \geqslant 0$ arbitrarily small. The main result consists of the full characterization of the $\eta$-NE information-energy region, i.e., the set of information-energy rate triplets $\left(R_{1}, R_{2}, B\right)$ that are achievable and stable in the G-MAC when: $(a)$ both transmitters autonomously and independently tune their own transmit configurations seeking to maximize their own information transmission rates, $R_{1}$ and $R_{2}$ respectively; (b) both transmitters jointly guarantee an energy transmission rate $B$ at the $\mathbf{E H}$, such that $B \geqslant b$. Therefore, any rate triplet outside the $\eta$-NE region is not stable as there always exists one transmitter able to increase by at least $\eta$ bits per channel use its own information transmission rate by updating its own transmit configuration.
\end{abstract}

\section{INTRODUCTION}

In wireless communication networks, energy and information can be simultaneously transmitted [1]. However, these two tasks are usually conflicting and thus, subject to a tradeoff between the information transmission rate (in bits per channel use) and the energy transmission rate (in energy units per channel use). In the discrete memoryless multiple access channel (MAC), this information-energy trade-off was studied by Fouladgar et al. [2]. Recently, Belhadj Amor et al. [3], [4] studied this problem in the Gaussian MAC (G-MAC) with and without feedback. In these works, the information-energy trade-off is modeled by the notion of information-energy capacity region, i.e., the set of all information-energy rate tuples that can be achieved. An object of central interest regarding this notion is that the achievability of these information-energy rate tuples is subject to the existence of a central controller that decides an operating point and indicates to all network components the corresponding transmit-receive configuration that should be used. Unfortunately, this assumption does not hold in networks in which a central controller is not feasible. This is typically the case of decentralized or ad hoc networks

Selma Belhadj Amor is with the Institut National de Recherche en Informatique et en Automatique (INRIA) at Lyon, France (selma.belhadjamor@inria.fr).

Samir M. Perlaza is with the Institut National de Recherche en Informatique et en Automatique (INRIA) at Lyon, France (samir.perlaza@inria.fr). He is also with the Department of Electrical Engineering at Princeton University, Princeton, NJ 08544, USA.

This research is supported in part by the European Commission under Marie Skłodowska-Curie Individual Fellowship No. 659316 (CYBERNETS). such as sensor networks, body area networks among others. In this type of multi-user channels, both transmitters and receivers are assumed to be autonomous and to be able of unilaterally choosing their own transmit-receive configurations aiming to maximize their individual benefit, e.g., individual information rate, individual energy rate or a combination of both. Hence, from this perspective, the notion of informationenergy capacity does not properly model the fundamental limits of simultaneous energy and information transmission (SEIT) in decentralized networks. To tackle this anarchical behavior observed in decentralized networks, the notion of stability is introduced and a new notion is presented: the energy-information $\eta$-Nash region. This region is the set of all information-energy rate tuples that are achievable and stable. In this case, stability is considered in the sense of an $\eta$-Nash equilibrium (NE) [5], with $\eta$ arbitrarily small. A multi-user channel is stable in the sense of an $\eta$-NE if none of the transmitters or receivers is able to increase its own individual benefit by more than $\eta$ units by unilaterally changing its transmit-receive configuration.

Previous works have studied decentralized MACs using game-theoretic tools when the aim of each transmitter is limited to exclusively transmitting information. For instance, Lai and El Gamal [6] proposed a framework to study the power allocation problem in fading decentralized MACs when transmitters aim to maximize their own individual transmission rate. Gajic and Rimoldi [7] considered a similar scenario with time-invariant channels in which transmitters have the choice of adopting any possible transmit configuration and determined the subregion of the information capacity region that is achievable at an NE. Varan and Yener [8] studied two-hop networks in which the source(s) is (are) incentivized to perform energy and signal cooperation to maximize the amount of its (their) own data that is reliably delivered to the destination.

This paper studies the fundamental limits of decentralized SEIT in the two-user G-MAC for the case in which a minimum energy rate is required for successful decoding. More specifically, each transmitter chooses its own transmit configuration aiming to maximize its individual information rate to the receiver while it guarantees an energy transmission rate higher than a given predefined threshold at a given energy harvester $(\mathrm{EH})$. The receiver is assumed to adopt a fixed configuration that can be either single-user decoding (SUD), successive interference cancellation (SIC) or any time-sharing configuration of the previous decoding techniques. The main contribution of this paper consists of the full characterization of the $\eta$-NE information-energy region of this multi-user 
channel, with $\eta$ arbitrarily small.

\section{Problem Formulation}

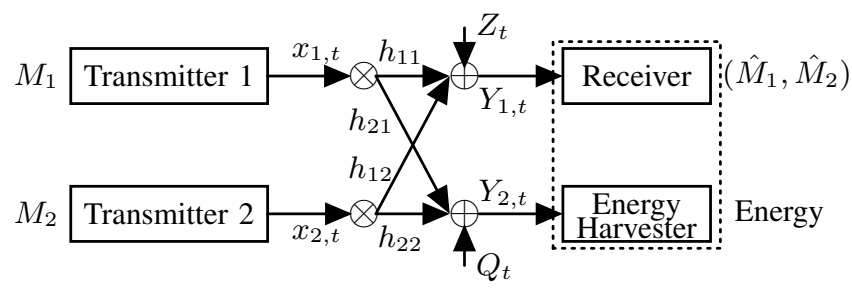

Fig. 1. Two-user memoryless Gaussian MAC with energy harvester.

\section{A. Channel Model}

Consider the two-user memoryless G-MAC with an EH as shown in Fig. 1. At each channel use $t \in \mathbb{N}, X_{1, t}$ and $X_{2, t}$ denote the real symbols sent by transmitters 1 and 2 , respectively. Let $n \in \mathbb{N}$ be the blocklength. The symbols $X_{i, 1}, \ldots, X_{i, n}$ satisfy an expected average input power constraint

$$
P_{i}=\frac{1}{n} \sum_{t=1}^{n} \mathrm{E}\left[X_{i, t}^{2}\right] \leqslant P_{i, \max },
$$

where $P_{i}$ and $P_{i, \max }$ denote respectively the average transmit power and the maximum average power of transmitter $i$ in energy units per channel use for $i \in\{1,2\}$. The receiver observes the real channel output

$$
Y_{1, t}=h_{11} X_{1, t}+h_{12} X_{2, t}+Z_{t},
$$

and the EH observes

$$
Y_{2, t}=h_{21} X_{1, t}+h_{22} X_{2, t}+Q_{t},
$$

where $h_{1 i}$ and $h_{2 i}$ are the corresponding constant non-negative channel coefficients from transmitter $i$ to the receiver and $\mathrm{EH}$, respectively. The channel coefficients satisfy the following $\mathcal{L}_{2}$ norm condition:

$$
\forall j \in\{1,2\}, \quad\left\|\mathbf{h}_{j}\right\|^{2} \leqslant 1,
$$

with $\mathbf{h}_{j} \triangleq\left(h_{j 1}, h_{j 2}\right)^{\top}$ in order to meet the energy conservation principle.

The signal to noise ratios (SNRs): $\mathrm{SNR}_{j i}$, with $\forall(i, j) \in$ $\{1,2\}^{2}$ are defined as follows

$$
\mathrm{SNR}_{j i} \triangleq\left|h_{j i}\right|^{2} P_{i, \text { max }},
$$

given the following normalization over the noise power. The noise terms $Z_{t}$ and $Q_{t}$ are realizations of two identically distributed zero-mean unit-variance real Gaussian random variables. In the following, there is no particular assumption on the joint distribution of $Q_{t}$ and $Z_{t}$. Within this context, two main tasks are to be simultaneously accomplished: information transmission and energy transmission.

\section{B. Information Transmission}

The goal of the communication is to convey the independent messages $M_{1}$ and $M_{2}$ from transmitter 1 and transmitter 2 to the common receiver. The message indices $M_{1}$ and $M_{2}$ are independent of the noise terms $Z_{1}, \ldots, Z_{n}, Q_{1}, \ldots, Q_{n}$ and uniformly distributed over the sets $\mathcal{M}_{1} \triangleq\left\{1, \ldots,\left\lfloor 2^{n R_{1}}\right\rfloor\right\}$ and $\mathcal{M}_{2} \triangleq\left\{1, \ldots,\left\lfloor 2^{n R_{2}}\right\rfloor\right\}$, where $R_{1}$ and $R_{2}$ denote the information transmission rates.

At each time $t$, the $t$-th symbol of transmitter $i$, for $i \in\{1,2\}$, depends solely on its message index $M_{i}$ and a randomly generated index $\Omega \in\left\{1, \ldots,\left\lfloor 2^{n R_{r}}\right\rfloor\right\}$, with $R_{r} \geqslant 0$, that is independent of both $M_{1}$ and $M_{2}$ and assumed to be known by all transmitters and the receiver, i.e.,

$$
X_{i, t}=f_{i, t}^{(n)}\left(M_{i}, \Omega\right), \quad t \in\{1, \ldots, n\},
$$

for some encoding functions $f_{i, t}^{(n)}: \mathcal{M}_{i} \times \mathbb{N} \rightarrow \mathbb{R}$. The receiver produces an estimate $\left(\hat{M}_{1}^{(n)}, \hat{M}_{2}^{(n)}\right)=\Phi^{(n)}\left(Y^{n}\right)$ of the message-pair $\left(M_{1}, M_{2}\right)$ via a decoding function $\Phi^{(n)}: \mathbb{R}^{n} \rightarrow$ $\mathcal{M}_{1} \times \mathcal{M}_{2}$, and the average probability of error is given by

$$
P_{\text {error }}^{(n)}\left(R_{1}, R_{2}\right) \triangleq \operatorname{Pr}\left\{\left(\hat{M}_{1}^{(n)}, \hat{M}_{2}^{(n)}\right) \neq\left(M_{1}, M_{2}\right)\right\} .
$$

\section{Energy Transmission}

The expected energy transmission rate (in energy units per channel use) at the EH is

$$
B^{(n)} \triangleq \frac{1}{n} \sum_{t=1}^{n} \mathrm{E}\left[Y_{2, t}^{2}\right] .
$$

The goal of the energy transmission is to guarantee that the expected energy rate $B^{(n)}$ is not less than a given target energy transmission rate $B$ that must satisfy

$$
0<B \leqslant 1+\mathrm{SNR}_{21}+\mathrm{SNR}_{22}+2 \sqrt{\mathrm{SNR}_{21} \mathrm{SNR}_{22}},
$$

for the problem to be feasible. Hence, the probability of energy outage is defined as follows:

$$
P_{\text {outage }}^{(n)}(B)=\operatorname{Pr}\left\{B^{(n)}<B-\epsilon\right\},
$$

for some $\epsilon>0$ arbitrarily small.

\section{Information-Energy Capacity Region}

The G-MAC in Fig. 1 is said to operate at the informationenergy rate triplet $\left(R_{1}, R_{2}, B\right) \in \mathbb{R}_{+}^{3}$ when both transmitters and the receiver use a transmit-receive configuration such that: (i) information transmission occurs at rates $R_{1}$ and $R_{2}$ with probability of error arbitrarily close to zero; and (ii) energy transmission occurs at a rate not smaller than $B$ with energy-outage probability arbitrarily close zero. Under these conditions, the information-energy rate triplet $\left(R_{1}, R_{2}, B\right)$ is said to be achievable.

Definition 1 (Achievable Rates). The triplet $\left(R_{1}, R_{2}, B\right) \in$ $\mathbb{R}_{+}^{3}$ is achievable if there exists a sequence of encoding and decoding functions $\left\{\left\{f_{1, t}^{(n)}\right\}_{t=1}^{n},\left\{f_{2, t}^{(n)}\right\}_{t=1}^{n}, \Phi^{(n)}\right\}_{n=1}^{\infty}$ such that both the average error probability and the energy-outage 
probability tend to zero as the blocklength $n$ tends to infinity. That is,

$$
\begin{aligned}
& \limsup _{n \rightarrow \infty} P_{\text {error }}^{(n)}\left(R_{1}, R_{2}\right)=0, \text { and } \\
& \limsup _{n \rightarrow \infty} P_{\text {outage }}^{(n)}(B) \quad=0 .
\end{aligned}
$$

Often, increasing the energy transmission rate implies decreasing the information transmission rates and vice versa. This trade-off is accurately modeled by the notion of information-energy capacity region.

Definition 2 (Information-Energy Capacity Region). The information-energy capacity region $\mathcal{E}\left(\mathrm{SNR}_{11}, \mathrm{SNR}_{12}, \mathrm{SNR}_{21}, \mathrm{SNR}_{22}\right)$ of the G-MAC is the closure of all achievable information-energy rate triplets $\left(R_{1}, R_{2}, B\right)$.

Lemma 1 (Theorem 1 in [3] ). The information-energy capacity region $\mathcal{E}\left(\mathrm{SNR}_{11}, \mathrm{SNR}_{12}, \mathrm{SNR}_{21}, \mathrm{SNR}_{22}\right)$ is the set of all non-negative information-energy rate triplets $\left(R_{1}, R_{2}, B\right)$ that satisfy

$$
\begin{aligned}
R_{1} \quad \leqslant & \frac{1}{2} \log _{2}\left(1+\beta_{1} \mathrm{SNR}_{11}\right), \\
R_{2} \leqslant & \frac{1}{2} \log _{2}\left(1+\beta_{2} \mathrm{SNR}_{12}\right) \\
R_{1}+R_{2} \leqslant & \frac{1}{2} \log _{2}\left(1+\beta_{1} \mathrm{SNR}_{11}+\beta_{2} \mathrm{SNR}_{12}\right), \\
B \leqslant & 1+\mathrm{SNR}_{21}+\mathrm{SNR}_{22} \\
& +2 \sqrt{\left(1-\beta_{1}\right) \mathrm{SNR}_{21}\left(1-\beta_{2}\right) \mathrm{SNR}_{22}},
\end{aligned}
$$

with $\left(\beta_{1}, \beta_{2}\right) \in[0,1]^{2}$.

From an achievability standpoint, the terms $\beta_{1}$ and $\beta_{2}$ in (13) might be interpreted as the fractions of power that transmitter 1 and transmitter 2 allocate for information transmission, respectively. The remaining fraction of power $\left(1-\beta_{i}\right)$ is allocated by transmitter $i$ for exclusively transmitting energy to the EH. More specifically, at each time $t$, transmitter $i$ 's channel input can be written as:

$$
X_{i, t}=\sqrt{\left(1-\beta_{i}\right) P_{i}} W_{t}+U_{i, t}, \quad i \in\{1,2\},
$$

for some independent zero-mean Gaussian informationcarrying symbols $U_{1, t}$ and $U_{2, t}$ with variances $\beta_{1} P_{1}$ and $\beta_{2} P_{2}$, respectively, and independent thereof $W_{t}$ are zero-mean unit-variance Gaussian energy-carrying symbols known noncausally to all terminals. The codebook and the encodingdecoding schemes for the information-carrying signals can be those described in [9] or [10]. Note that the common randomness carries energy to the EH but does not carry any information to the receiver. Indeed, it does not produce any interference to the information-carrying signals as its effect can be suppressed at the receiver using classical SIC.

\section{Gaussian MAC with Minimum Energy Rate $b$}

Consider a G-MAC in which reliable decoding is subject to the classical conditions of the G-MAC [9], [10] and an additional condition:

$$
\limsup _{n \rightarrow \infty} P_{\text {outage }}^{(n)}(b)=0,
$$

that is, the rate at which energy is collected at a given point, e.g., an $\mathrm{EH}$, is not less than a given target energy transmission rate $b$ which satisfies the following:

$$
0 \leqslant b \leqslant 1+\mathrm{SNR}_{21}+\mathrm{SNR}_{22}+2 \sqrt{\mathrm{SNR}_{21} \mathrm{SNR}_{22}} .
$$

That is, in this scenario both transmitters simultaneously transmit energy to an $\mathrm{EH}$ and information to the receiver. Note that in the case in which $b \in\left[0,1+\min \left(\mathrm{SNR}_{21}, \mathrm{SNR}_{22}\right)\right]$, both transmitters, independently of each other can always use a coding scheme as the one in (14) in which all the power budget is used for generating information-carrying signals at a strictly positive information rate and ensure reliable decoding. In this case, the minimum energy constraint does not play a fundamental role. Alternatively, when $b \in\left[1+\min \left(\mathbf{S N R}_{21}, \mathbf{S N R}_{22}\right), 1+\max \left(\mathbf{S N R}_{21}, \mathbf{S N R}_{22}\right)\right]$, reliable decoding of the messages of the transmitter with the lowest SNR, say transmitter 1, depends on whether or not transmitter 2 is sending messages using an average power such that $\lim _{n \rightarrow \infty} B^{(n)} \geqslant b$ at least with probability arbitrarily close to 1 . Conversely, transmitter 2 can always use a coding scheme as the one in (14) in which all the power budget is used for generating information-carrying signals at a strictly positive information rate and ensure reliable decoding. In this case, the minimum energy rate constraint drastically affects the way that both transmitters interact with each other. More critical scenarios are the case in which $\left.b \in\left(1+\max \left(\mathbf{S N R}_{21}, \mathbf{S N R}_{22}\right)\right], 1+\mathbf{S N R}_{21}+\mathbf{S N R}_{22}\right]$ and $b \in$ $\left(1+\mathrm{SNR}_{21}+\mathrm{SNR}_{22}, 1+\mathrm{SNR}_{21}+\mathrm{SNR}_{22}+2 \sqrt{\mathrm{SNR}_{21} \mathrm{SNR}_{22}}\right.$. In these cases, none of the transmitters can unilaterally ensure reliable decoding. In the former, having both transmitters use all their power budget to generate information-carrying signals at a strictly positive rate ensures reliable decoding of the messages of both transmitters. In the latter, this strategy is no longer sufficient and thus, both transmitters must engage in a mechanism through which an energy rate that is higher than the energy delivered by exclusively transmitting informationcarrying signals is ensured at the EH. This suggests for instance, the use of power-splits in which the transmitted symbols have an information-carrying and an energy-carrying components as in (14).

This problem can be studied from a centralized or decentralized point of view. In the former, there exists a central controller that determines an operating point and indicates to each transmitter and the receiver the corresponding transmitreceive configuration to achieve such a point. In the latter, each network component is considered to be autonomous and seeks to determine its own transmit-receive configuration in order to maximize its individual benefit, e.g., its own information transmission rate.

\section{A. Centralized Case}

Let $b \geqslant 0$ denote the minimum energy rate that must be guaranteed at the input of the EH in the G-MAC. Hence, the set of all information-energy rate triplets that are achievable is fully described by the following theorem.

Theorem 1 (Information-Energy Capacity Region with Minimum Energy Constraint). Let $b \in\left[0,1+\mathbf{S N R}_{21}+\mathrm{SNR}_{22}+\right.$ 
$\left.2 \sqrt{\mathrm{SNR}_{21} \mathrm{SNR}_{22}}\right]$. Then, the information-energy capacity region $\mathcal{E}_{b}\left(\mathrm{SNR}_{11}, \mathrm{SNR}_{12}, \mathrm{SNR}_{21}, \mathrm{SNR}_{22}\right)$ with minimum energy constraint $b$ is the set of non-negative triplets $\left(R_{1}, R_{2}, B\right) \in$ $\mathcal{E}\left(\mathrm{SNR}_{11}, \mathrm{SNR}_{12}, \mathrm{SNR}_{21}, \mathrm{SNR}_{22}\right)$ that satisfy $B \geqslant b$.

Proof: The proof of Theorem 1 follows immediately from [3, Proposition 1] and [3, Theorem 2].

The maximum individual rate given a minimum energy transmission rate $b$ is

$$
R_{i, \max }(b)=\frac{1}{2} \log _{2}\left(1+\gamma(b) \mathrm{SNR}_{1 i}\right),
$$

with $\gamma(b)=1-\left(\frac{\left(b-\left(1+\mathrm{SNR}_{21}+\mathrm{SNR}_{22}\right)\right)^{+}}{2 \sqrt{\mathrm{SNR}_{21} \mathrm{SNR}_{22}}}\right)^{2}$. This rate is achieved by transmitter $i$, for instance, when transmitter $j$ is using all its available power $\left(\beta_{j}=0\right)$ for exclusively transmitting energy to the EH by using common randomness; and transmitter $i$ is using a power-split in which the part of power dedicated for exclusively transmitting energy to the $\mathrm{EH}$, $1-\beta_{i}$, is the fraction needed to satisfy

$1+\mathrm{SNR}_{21}+\mathrm{SNR}_{22}+2 \sqrt{\left(1-\beta_{1}\right) \mathrm{SNR}_{21}\left(1-\beta_{2}\right) \mathrm{SNR}_{22}} \geqslant b$,

with equality, that is, $\beta_{i}=\gamma(b)$ (when $\beta_{j}=0$ ). The maximum information sum-rate is

$$
R_{\text {sum }, \max }(b)=\frac{1}{2} \log _{2}\left(1+\alpha(b)\left(\operatorname{SNR}_{11}+\operatorname{SNR}_{12}\right)\right),
$$

with $\alpha(b)=\min \left\{1,\left(\frac{1+\mathrm{SNR}_{21}+\mathrm{SNR}_{22}+2 \sqrt{\mathrm{SNR}_{21} \mathrm{SNR}_{22}}-b}{2 \sqrt{\mathrm{SNR}_{21} \mathrm{SNR}_{22}}}\right)^{+}\right\}$. This information sum-rate is achievable for instance when both transmitters use a power-split $\left(\beta_{1}, \beta_{2}\right)$ that satisfies (18) with equality $\left(\beta_{1}=\beta_{2}=\alpha(b)\right)$ and the receiver implements successive interference cancellation.

\section{B. Decentralized Case}

Consider a decentralized G-MAC in which the aim of transmitter $i$, for $i \in\{1,2\}$, is to autonomously choose its transmit configuration $s_{i}$ in order to maximize its information rate $R_{i}$, while guaranteeing a minimum energy rate $b$ at the EH (see (15)). In particular, the transmit configuration $s_{i}$ can be described in terms of the information rates $R_{i}$, the blocklength $n$, the channel input alphabet $\mathcal{X}_{i}$, the encoding functions $f_{i}^{(1)}, \ldots, f_{i}^{(n)}$, the common randomness, the power dedicated to information and energy transmission, etc. The receiver is assumed to adopt a fixed decoding strategy that is known in advance by all transmitters.

Note that if the aim of each transmitter, say transmitter $i$, is to maximize its own individual information rate $R_{i}$ subject to the minimum energy rate $b$ at the $\mathrm{EH}$, it is clear from (13) that one option should be using a power-split in which the component dedicated to the transmission of information $\beta_{i}$ is as high as possible. However, its power-split $\beta_{i}$ must also be chosen such that the energy-outage probability (15) can be made arbitrarily close to zero.

This reveals that the choice of the transmit configuration of each transmitter is subject to each other as both transmitters must guarantee the minimum energy constraint required at the $\mathrm{EH}$; and at the same time, depending on the decoding scheme at the receiver, the information-carrying signal of one transmitter is interference to the other transmitter. This reasoning implies that the rate achieved by transmitter $i$ depends on both configurations $s_{1}$ and $s_{2}$ as well as the configuration of the receiver, even if it is assumed to be fixed. This justifies the analysis of this scenario using tools from game theory.

\section{Game Formulation}

The competitive interaction of the two transmitters and the receiver in the decentralized Gaussian MAC with minimum energy constraint $b$ described in Sec. III-B can be modeled by the following game in normal form:

$$
\mathcal{G}(b)=\left(\mathcal{K},\left\{\mathcal{A}_{k}\right\}_{k \in \mathcal{K}},\left\{u_{k}\right\}_{k \in \mathcal{K}}\right),
$$

where $b$ is a parameter of the game that represents the minimum energy-rate that must be guaranteed at the $\mathrm{EH}$ (see (15)). The set $\mathcal{K}=\{1,2\}$ is the set of players, that is, transmitter 1 and transmitter 2 . The sets $\mathcal{A}_{1}$ and $\mathcal{A}_{2}$ are the sets of actions of players 1 and 2 , respectively. An action of a player $i \in \mathcal{K}$, which is denoted by $s_{i} \in \mathcal{A}_{i}$, is basically its transmit configuration as described above. The utility function of transmitter $i$, for $i \in\{1,2\}$, is $u_{i}: \mathcal{A}_{1} \times \mathcal{A}_{2} \rightarrow \mathbb{R}_{+}$and it is defined as its own information rate,

$$
u_{i}\left(s_{1}, s_{2}\right)=\left\{\begin{array}{lll}
R_{i}\left(s_{1}, s_{2}\right), & \text { if } \quad \begin{array}{l}
P_{\text {error }}^{(n)}<\epsilon \text { and } P_{\text {outage }}^{(n)}<\delta \\
-1,
\end{array} \quad \text { otherwise },
\end{array}\right.
$$

where $\epsilon>0$ and $\delta>0$ are arbitrarily small numbers and $R_{i}\left(s_{1}, s_{2}\right)$ denotes an information transmission rate achievable (Def. 1) with the configurations $s_{1}$, and $s_{2}$. Often, the information rate $R_{i}\left(s_{1}, s_{2}\right)$ is written as $R_{i}$ for simplicity. However, every non-negative achievable information rate is associated with a particular transmit-receive configuration pair $\left(s_{1}, s_{2}\right)$ that achieves it. It is worth noting that there might exist several transmit-receive configurations that achieve the same triplet $\left(R_{1}, R_{2}, B\right)$ and distinction between the different transmitreceive configurations is made only when needed.

A class of transmit-receive configurations $s^{*}=\left(s_{1}^{*}, s_{2}^{*}\right) \in$ $\mathcal{A}_{1} \times \mathcal{A}_{2}$ that are particularly important in the analysis of this game are referred to as $\eta$-Nash equilibria ( $\eta$-NE).

\section{A. $\eta$-Nash Equilibrium}

A transmit-receive configuration $s^{*}=\left(s_{1}^{*}, s_{2}^{*}\right) \in \mathcal{A}_{1} \times \mathcal{A}_{2}$ that is an $\eta$-NE satisfies the following conditions:

Definition 3 ( $\eta$-NE [11]). In the game $\mathcal{G}(b)=$ $\left(\mathcal{K},\left\{\mathcal{A}_{k}\right\}_{k \in \mathcal{K}},\left\{u_{k}\right\}_{k \in \mathcal{K}}\right)$, an action profile $\left(s_{1}^{*}, s_{2}^{*}\right)$ is an $\eta$ $\mathrm{NE}$ if for all $\left(s_{1}, s_{2}\right) \in \mathcal{A}_{1} \times \mathcal{A}_{2}$, it holds that

$$
\begin{aligned}
& u_{1}\left(s_{1}, s_{2}^{*}\right) \leqslant u_{1}\left(s_{1}^{*}, s_{2}^{*}\right)+\eta, \text { and } \\
& u_{2}\left(s_{1}^{*}, s_{2}\right) \leqslant u_{2}\left(s_{1}^{*}, s_{2}^{*}\right)+\eta .
\end{aligned}
$$

From Def. 3 , it becomes clear that if $\left(s_{1}^{*}, s_{2}^{*}\right)$ is an $\eta$-NE, then none of the transmitters can increase its own information transmission rate by more than $\eta$ bits per channel use by changing its own transmit-receive configuration and keeping the average error probability and the energy outage probability arbitrarily close to zero. Thus, at a given $\eta$-NE, every player achieves a utility that is $\eta$-close to its maximum achievable rate given the transmit-receive configuration of the other players. 
Note that if $\eta=0$, then the classical definition of $\mathrm{NE}$ is obtained [5]. The following investigates the set of information and energy rate triplets that can be achieved at an $\eta$-NE. This set of rate triplets is known as the $\eta$-NE information-energy region.

Definition 4 ( $\eta$-NE Region). Let $\eta \geqslant 0$. An achievable information-energy rate triplet $\left(R_{1}, R_{2}, B\right) \in$ $\mathcal{E}_{b}\left(\mathrm{SNR}_{11}, \mathrm{SNR}_{12}, \mathrm{SNR}_{21}, \mathrm{SNR}_{22}\right)$ is said to be in the $\eta$-NE region of the game $\mathcal{G}(b)=\left(\mathcal{K},\left\{\mathcal{A}_{k}\right\}_{k \in \mathcal{K}},\left\{u_{k}\right\}_{k \in \mathcal{K}}\right)$ if there exists a triplet $\left(s_{1}^{*}, s_{2}^{*}\right) \in \mathcal{A}_{1} \times \mathcal{A}_{2}$ that is an $\eta$-NE and the following holds:

$$
\begin{aligned}
& u_{1}\left(s_{1}^{*}, s_{2}^{*}\right)=R_{1} \text { and } \\
& u_{2}\left(s_{1}^{*}, s_{2}^{*}\right)=R_{2}
\end{aligned}
$$

The following section studies the $\eta$-NE region of the game $\mathcal{G}(b)$, with $\eta \geqslant 0$ arbitrarily small.

\section{Main Results}

For a fixed energy transmission rate $b \geqslant 0$ required at the input of the $\mathrm{EH}$ (see (15)), let the set $\mathcal{D}(b)$ be defined as follows:

$$
\begin{aligned}
& \mathcal{D}(b)=\left\{\left(\beta_{1}, \beta_{2}\right) \in[0,1]^{2}:\right. \\
& \left.\sqrt{\left(1-\beta_{1}\right)\left(1-\beta_{2}\right)}=\frac{\left(b-\left(1+\mathrm{SNR}_{21}+\mathrm{SNR}_{22}\right)\right)^{+}}{2 \sqrt{\mathrm{SNR}_{21} \mathrm{SNR}_{22}}}\right\} .
\end{aligned}
$$

The $\eta$-NE region of the game $\mathcal{G}(b)$ when the receiver uses single-user decoding (SUD), denoted by $\mathcal{N}_{\mathrm{SUD}}(b)$, is described by the following theorem.

Theorem 2 ( $\eta$-NE Region of the Game $\mathcal{G}(b)$ with SUD). Let $b \in\left[0,1+\mathrm{SNR}_{21}+\mathrm{SNR}_{22}+2 \sqrt{\mathrm{SNR}_{21} \mathrm{SNR}_{22}}\right]$ and $\eta \geqslant 0$ arbitrarily small. Then, the set $\mathcal{N}_{\mathrm{SUD}}(b)$ of $\eta$-NEs of the game $\mathcal{G}(b)$ is defined as follows:

$$
\begin{aligned}
\mathcal{N}_{\mathrm{SUD}}(b) & =\left\{\left(R_{1}, R_{2}, B\right) \in \mathbb{R}_{+}^{3}:\left(\beta_{1}, \beta_{2}\right) \in \mathcal{D}(b)\right. \text { and } \\
R_{1} & =\frac{1}{2} \log _{2}\left(1+\frac{\beta_{1} \mathrm{SNR}_{11}}{1+\beta_{2} \mathrm{SNR}_{12}}\right) \\
R_{2} & =\frac{1}{2} \log _{2}\left(1+\frac{\beta_{2} \mathrm{SNR}_{12}}{1+\beta_{1} \mathrm{SNR}_{11}}\right) \\
B & \geqslant b \text { and } \\
B & \leqslant 1+\mathrm{SNR}_{21}+\mathrm{SNR}_{22} \\
+ & \left.\sqrt{\left(1-\beta_{1}\right) \mathrm{SNR}_{21}\left(1-\beta_{2}\right) \mathrm{SNR}_{22}} \cdot\right\}
\end{aligned}
$$

Proof: The proof of Theorem 2 is presented in [12].

Let $\operatorname{SIC}(i \rightarrow j)$ denote the case in which the receiver uses successive interference cancellation (SIC) with decoding order: transmitter $i$ before transmitter $j$, with $i \in\{1,2\}$. Then, the $\eta$-NE region of the game $\mathcal{G}(b)$ when the receiver uses $\mathrm{SIC}(i \rightarrow j)$, denoted by $\mathcal{N}_{\mathrm{SIC}(i \rightarrow j)}(b)$, is described by the following theorem.

Theorem 3 ( $\eta$-NE Region of the Game $\mathcal{G}(b)$ with SIC). Let $b \in\left[0,1+\mathrm{SNR}_{21}+\mathrm{SNR}_{22}+2 \sqrt{\mathrm{SNR}_{21} \mathrm{SNR}_{22}}\right]$ and $\eta \geqslant 0$ arbitrarily small. Then, the set $\mathcal{N}_{\mathrm{SIC}(i \rightarrow j)}(b)$ is defined as follows:

$$
\begin{aligned}
\mathcal{N}_{\mathrm{SIC}(i \rightarrow j)}(b) & =\left\{\left(R_{1}, R_{2}, B\right) \in \mathbb{R}_{+}^{3}:\left(\beta_{1}, \beta_{2}\right) \in \mathcal{D}(b)\right. \text { and } \\
R_{i} & =\frac{1}{2} \log _{2}\left(1+\frac{\beta_{i} \mathrm{SNR}_{1 i}}{1+\beta_{j} \mathrm{SNR}_{1 j}}\right) \\
R_{j} & =\frac{1}{2} \log _{2}\left(1+\beta_{j} \mathrm{SNR}_{1 j}\right) \\
B & \geqslant b \text { and } \\
B & \leqslant 1+\mathrm{SNR}_{21}+\mathrm{SNR}_{22} \\
+ & \left.\sqrt{\left(1-\beta_{1}\right) \mathrm{SNR}_{21}\left(1-\beta_{2}\right) \mathrm{SNR}_{22}}\right\}
\end{aligned}
$$

Proof: The proof of Theorem 3 is presented in [12].

Before discussing the insights arising from Theorem 2 Theorem 3, consider a symmetric G-MAC with $\mathrm{SNR}_{11}=$ $\mathrm{SNR}_{12}=\mathrm{SNR}_{21}=\mathrm{SNR}_{22}=10(\mathrm{EH}$ and receiver are co-located). Note that for all $b \leqslant 1+\mathrm{SNR}_{21}+\mathrm{SNR}_{22}$, all transmitters use the whole available average power for information transmission as shown in Fig. 2. Alternatively, when $b>1+\mathrm{SNR}_{21}+\mathrm{SNR}_{22}$, both transmitters use the minimum energy needed to make the energy-outage probability arbitrarily close to zero and seek for the largest possible information transmission rate (See Fig. 3).

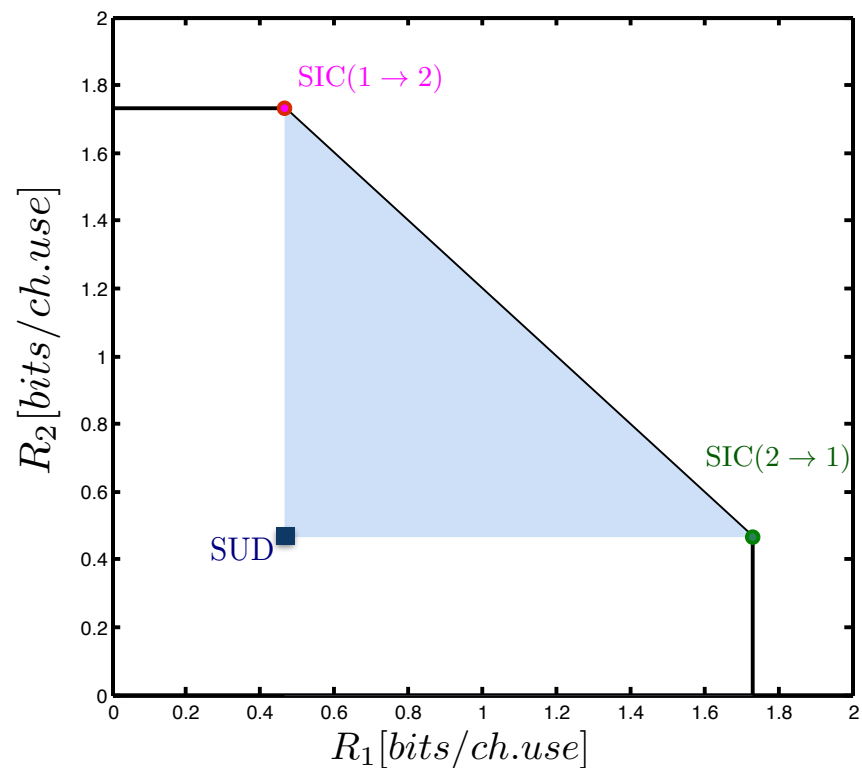

Fig. 2. Projection of the set $\mathcal{N}_{\mathrm{SUD}}(b)$ (square point) and $\mathcal{N}_{\mathrm{SIC}(i \rightarrow j)}(b)$ (round points) over the $R_{1}-R_{2}$ plane for $b \leqslant 1+\mathrm{SNR}_{21}+\mathrm{SNR}_{22}$. The information capacity region is also plotted as a reference (white region inside solid lines) for $\mathrm{SNR}_{11}=\mathrm{SNR}_{12}=\mathrm{SNR}_{21}=\mathrm{SNR}_{22}=10$. Note that the information capacity region with and without energy transmission rate constraint are identical in this case. The blue region is the convex hull of $\mathcal{N}_{\mathrm{SUD}}(b) \cup \mathcal{N}_{\mathrm{SIC}(1 \rightarrow 2)}(b) \cup \mathcal{N}_{\mathrm{SIC}(2 \rightarrow 1)}(b)$.

\section{DISCUSSION}

1) Existence of an $\eta$-NE: One of the main observations is that the existence of an $\eta$-NE, with $\eta$ arbitrarily small, is always guaranteed as long as the SEIT problem is feasible, 


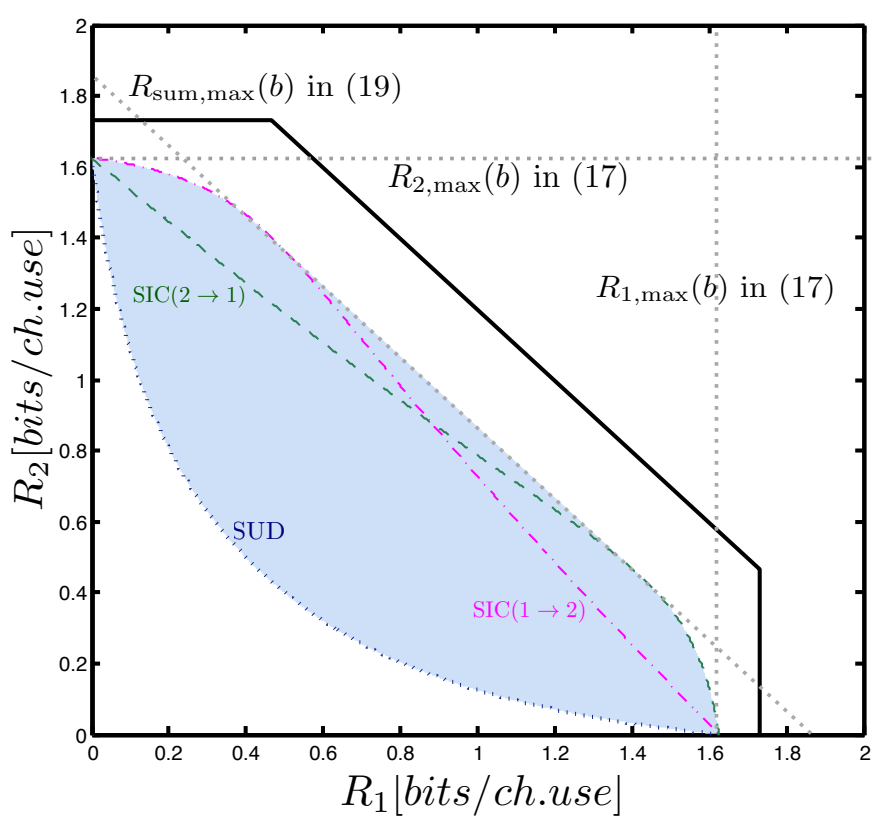

Fig. 3. Projection of the set $\mathcal{N}_{\mathrm{SUD}}(b)$ (dotted line) and $\mathcal{N}_{\mathrm{SIC}(i \rightarrow j)}(b)$ (dashed lines) over the $R_{1}-R_{2}$ plane for $b=0.7 B_{\max }>1+\mathrm{SNR}_{21}+$ $\mathrm{SNR}_{22}$. The information capacity region without energy transmission constraints (region inside solid lines) is plotted for $\mathrm{SNR}_{11}=\mathrm{SNR}_{12}=\mathrm{SNR}_{21}=$ $\mathrm{SNR}_{22}=10\left(\right.$ Note that $\left.B_{\max } \triangleq 1+\mathrm{SNR}_{21}+\mathrm{SNR}_{22}+2 \sqrt{\mathrm{SNR}_{21} \mathrm{SNR}_{22}}\right)$. The blue region is the convex hull of $\mathcal{N}_{\mathrm{SUD}}(b) \cup \mathcal{N}_{\mathrm{SIC}(1 \rightarrow 2)}(b) \cup$ $\mathcal{N}_{\mathrm{SIC}(2 \rightarrow 1)}(b)$.

that is, $b \leqslant 1+\mathrm{SNR}_{21}+\mathrm{SNR}_{22}+2 \sqrt{\mathrm{SNR}_{21} \mathrm{SNR}_{22}}$. This statement follows immediately from the fact that $\mathcal{N}(b) \neq \emptyset$, which ensures the existence of at least one action profile $\left(s_{1}^{*}, s_{2}^{*}\right)$ that is an $\eta$-NE. Interestingly, when $b>1+\mathrm{SNR}_{21}+$ $\mathrm{SNR}_{22}+2 \sqrt{\mathrm{SNR}_{21} \mathrm{SNR}_{22}}$, the energy transmission cannot be performed reliably and thus, the information-energy capacity region is empty and so is the $\eta$-NE region. However, this does not imply that there does not exist at least one $\eta$-NE in this particular case.

Remark: Note that for any given $b \geqslant 0$, the sets $\mathcal{N}_{\mathrm{SUD}}(b)$ and $\mathcal{N}_{\text {SIC }}(b)$ include only those information-energy triples $\left(R_{1}, R_{2}, B\right)$ that satisfy $B \geqslant b$. That is, the $\eta$-NEs at which the energy constraint can be satisfied. However, this suggests that there might exists other $\eta$-NE that are not in these sets at which either one of the conditions either (11) or (12) are not met. Consider for instance a case in which $b \geqslant 1+\max \left(\mathrm{SNR}_{11}, \mathrm{SNR}_{12}\right)$ and both transmitters decide to use the strategies $s_{1}$ and $s_{2}$ at which none of the transmitters actually transmits, e.g., standby mode. Hence, none of the transmitters can unilaterally deviate and achieve a utility other than $u_{1}\left(a_{1}, a_{2}\right)=u_{2}\left(a_{1}, a_{2}\right)=-1$ which translates into an information-energy triplet $(0,0,0)$ which is also an $\eta$-NE but is not in any of the sets $\mathcal{N}_{\mathrm{SUD}}(b)$ or $\mathcal{N}_{\mathrm{SIC}(\mathrm{i} \rightarrow \mathrm{j})}(b)$ as the energy constraint cannot be satisfied (Def. 4).

2) Cardinality of the set of $\eta-N E$ equilibria: The unicity of a given $\eta$-NE of the game $\mathcal{G}(b)$ is not ensured even in the case in which the cardinality of the $\eta$-NE informationenergy region is one. Consider the case in which $\eta=0$ and $b=1+\mathrm{SNR}_{21}+\mathrm{SNR}_{22}+2 \sqrt{\mathrm{SNR}_{21} \mathrm{SNR}_{22}}$. In this case, $\mathcal{N}(b)=\left\{\left(0,0,1+\mathrm{SNR}_{21}+\mathrm{SNR}_{22}+2 \sqrt{\mathrm{SNR}_{21} \mathrm{SNR}_{22}}\right)\right\}$ and for instance, using all the power budget for sending common randomness is an NE action profile. However, there is an infinite number of possible common random sequences that can be adopted by both transmitters independently of the action taken by the receiver as in this case $R_{1}=R_{2}=0$. The cardinality of the set of $\eta$-NEs is an acceptable lowerbound for the number of equilibria. This suggests that if the cardinality of the $\eta$-NE set is infinity, hence the number of $\eta$-NE is also infinity as every information-energy rate triplet in $\mathcal{N}(b)$ is associated with at least one achievability scheme that is an $\eta$-NE (Def. 4).

3) Optimality of the $\eta$-NE: Probably the most interesting observation regarding Theorem 2 and Theorem 3 is that some of the sum-rate optimal triples $\left(R_{1}, R_{2}, B\right)$ given a minimum energy-rate $b$ required at the $\mathrm{EH}$ are achievable at an $\eta$-NE. These $\eta$-NE sum-rate optimal triplets are Pareto optimal points of the information-energy capacity region $\mathcal{E}\left(\mathrm{SNR}_{11}, \mathrm{SNR}_{12}, \mathrm{SNR}_{21}, \mathrm{SNR}_{22}\right)$. This suggests that, under the assumption that players are able to properly choose the operating $\eta$-NE for instance via learning algorithms, there is no loss of performance in the decentralized SEIT case with respect to the fully centralized SEIT case.

\section{REFERENCES}

[1] L. R. Varshney, "On energy/information cross-layer architectures," in Proc. IEEE International Symposium on Information Theory, Jul. 2012, pp. 1356-1360.

[2] A. M. Fouladgar; and O. Simeone, "On the transfer of information and energy in multi-user systems," IEEE Communications Letters, vol. 16, no. 11, pp. 1733-1736, Nov. 2012.

[3] S. Belhadj Amor, S. M. Perlaza, I. Krikidis, and H. V. Poor, "Feedback enhances simultaneous information and energy transmission in multiple access channels," INRIA, Lyon, France, Tech. Rep. RR-8804, Nov. 2015.

[4] S. Belhadj Amor, S. M. Perlaza, and I. Krikidis, "Simultaneous energy and information transmission in Gaussian multiple access channels," in Proc. 5th International Conference on Communications and Networking (ComNet), Hammamet, Tunisia, Nov. 2015.

[5] J. F. Nash, "Equilibrium points in $n$-person games," Proc. of the National Academy of Sciences, vol. 36, pp. 48-49, 1950.

[6] L. Lai and H. El Gamal, "The water-filling game in fading multipleaccess channels," IEEE Transactions on Information Theory, vol. 54, no. 5, pp. 2110-2122, May 2008.

[7] V. Gajic and B. Rimoldi, "Game theoretic considerations for the Gaussian multiple access channel," in Proc. IEEE International Symposium on Information Theory, July 2008, pp. 2523-2527.

[8] B. Varan and A. Yener, "Incentivizing signal and energy cooperation in wireless networks," IEEE Journal on Selected Areas in Communications, vol. 33, no. 12, pp. 2554-2566, Dec 2015.

[9] T. M. Cover, Some Advances in Broadcast Channels. Academic Press, 1975, vol. 4, ch. 4

[10] A. D. Wyner, "Recent results in the Shannon theory," IEEE Transactions on Information Theory, vol. 20, no. 1, pp. 2-10, 1974.

[11] N. Nisan, T. Roughgarden, E. Tardos, and V. V. Vazirani, Algorithmic game theory. Cambridge, New York, Melbourne: Cambridge University Press, 2007.

[12] S. Belhadj Amor and S. M. Perlaza, "Decentralized simultaneous energy and information transmission in multiple access channels," INRIA, Lyon, France, Tech. Rep. RR-8847, Jan. 2016. 